\title{
Human Nature in a Post-Essentialist World
}

\author{
Grant Ramsey \\ Department of Philosophy \\ University of Notre Dame
}

1. Introduction. In a Platonic worldview, which species an individual belongs to is answered by this question: in which form does it participate? A goat is a goat because it participates in the Platonic form goat and not, say, the form horse. The tidiness of this answer, however, belies the questionable ontology on which it rests. It is for this reason that contemporary philosophers generally eschew the invocation of Platonic forms in producing a theory of species natures.

Nevertheless, species seem to have a nature-perhaps not one founded on Platonic forms, but instead on a set of essential properties. If we were given a lineup of a random assortment of vertebrates_-wildebeest, aardvarks, naked mole rats, vampire bats, and humans — we would have no problem picking out the humans. Even if the lineup were populated with our closest living relatives, we would have no difficulty picking the humans out from the other apes. This is true in part because humans differ in many ways from other species of ape. But it is not merely that humans differ from the other related species, it is also that individual humans share many traits amongst themselves. This similarity - this set of traits that it seems we are able register intuitively to instantly recognize an individual as a human being —is, it would seem, what we could use as a respectable foundation for human nature: human nature is just that set of traits that are possessed by to each individual and essential to their being human.

But with Darwin's (1859) publication of On the Origin of Species, an essentialist view of species was called into question. Darwin argued that the history of life has a tree 
structure and that branches on this tree represent biological taxa. A large branch represents a high-level taxon, such as a class or phylum, whereas a small branch represents a small taxon like a genus or species. For Darwin, species are not ontologically sui generis. Instead, there is a continuum from variations within species to genuine specieshood. On this view, taxonomists debate the number of species within a taxonflowering plants, for example — not (merely) because there is a dearth of taxonomic data, but because there are (often well-justified) differing approaches to drawing a line in the continuum between variations and species.

Darwin's view of species and the origin of species through intraspecific variation is mirrored by contemporary biology. The question at hand, then, is whether the concept of species nature - and in particular human nature - has any place in this contemporary theoretical context. In what follows, I will examine two ways in which philosophers have answered this question. I will then show that neither answer is satisfactory and will then present my own alternative.

2. Hull's skepticism about human nature. The main line contemporary taxonomic framework, cladistics, recognizes and formalizes Darwin's insights about the tree structure of life. For cladists, the only legitimate taxa are monophyletic clades, which are groups formed by encircling an ancestor and all of the branches descended from this ancestor. Taxonomic groupings that include multiple clades (polyphyletic groups) or fail to include all of the branches within a clade (paraphyletic groups) are not legitimate taxa. Thus, a species must be monophyletic, and not polyphyletic or paraphyletic. A corollary 
of these restrictions is that in order for a new taxon to arise, there must be a branching event. No new taxa, no new species, genera, etc., can arise in the absence of branching. It is this aspect of contemporary taxonomy that Hull (1986) uses to make his argument that there is no such thing as human nature. Hull's arguments can be summed up in the following way: 'Human nature' must pick out intrinsic traits that are exhibited by all (and only) humans. This set of traits must be definitive of and essential to membership in the species Homo sapiens, just as having eight protons is definitive of and essential to being oxygen. But membership in Homo sapiens, as with membership in any biological species is determined not by essential properties shared by each individual, but instead by their position within a clade. Thus, such essential properties cannot be definitive of species membership. Additionally, these properties are unlikely to be exhibited by all Homo Sapiens (individuals with severe developmental disorders are members of our species, after all). Further, even if a synchronic time slice of Homo sapiens reveals an interesting set of shared traits, the species will keep evolving and these traits are unlikely to persist over the entire existence of the species.

One way to understand Hull's argument is that he takes a species to be an individual whose birth and death are marked by phylogenetic branching events (nodes). The organisms belonging to the species are thus all of the organisms existing between the nodes. Hull's synchronic argument says that a synchronic slice in this species is unlikely to produce a set of individuals with interesting traits shared by them and only them. And the diachronic argument goes further to say that even if the synchronic slice produces interesting traits, these traits are probably not going to persist through all intra-nodal time. 
3. Machery's reply and its limitations. In the face of Hull's skepticism, one could either concede that there really is no such thing as human nature, or produce a concept of human nature that eschews or challenges Hull's criticisms. Machery (2008) attempts the latter. He argues that while Hull's arguments are devastating to essentialist notions of human nature, they do not undermine another concept of human nature, what he is labeling the "nomological" view. Machery's nomological view holds that "human nature is the set of properties that humans tend to possess as a result of the evolution of their species" (323).

Machery's account addresses Hull's worries in part by no longer considering human nature to be definitional. Thus, because an individual is not defined as being a human, that is, belonging to the species Homo sapiens, in virtue of possessing the traits that fall under the rubric of human nature, particular individuals can lack one or more of these traits while still being human. Instead, Machery merely requires that any trait considered part of human nature must be possessed by most humans.

Despite the successful dodge of Hull's arguments, there are difficulties with Machery's view. First, by requiring that the trait be possessed by the majority of humans, one looses many traits characteristic of humans. Any traits (psychological, behavioral, morphological) that are sexually dimorphic or, say, exhibited only by a particular ethnic group, will be excluded. Vivipary, lactation, and menopause, for example, are no part of human nature. Machery recognizes this, but holds to his view because "saying that humans have a nature entails that humans form a class that is of importance for biology. 
The members of this class tend to have some properties in common in virtue of evolutionary processes" (326).

But why does belonging to almost all humans make it an important class for biology? Is it not a biologically interesting feature of human nature that the females undergo menopause? Furthermore, why should we presume that it is the sameness across individuals that is of interest to scientists, and not their variation? As Geertz (1973) insightfully pointed out, "[t]he notion that unless a cultural phenomenon is empirically universal it cannot reflect anything about the nature of man is about as logical as the notion that because sickle-cell anemia is, fortunately, not universal, it cannot tell us anything about human genetic processes. It is not whether phenomena are empirically common that is critical in science $[\ldots]$ but whether they can be made to reveal the enduring natural processes that underly them" (44). I am in full agreement with Geertz on this point — it a mistake to hold that only traits universal (or nearly universal) in the human species are of scientific interest and should be included within human nature.

The second difficulty is that Machery takes it to be unproblematic to sort properties into two bins, those due to "the evolution of their species" (323) and those "exclusively due to enculturation or to social learning" (326). Only the former, asserts Machery, are a part of human nature. But what, exactly, is a property "exclusively due to enculturation or to social learning"? Any organismic property is going to be due both to heritable features of the organism as well as the particular environmental features the organism happens to encounter during its life. Some of these environmental features could be counted as instances of "enculturation" or "social learning," but the fact that such environmental features are present in the organism's life history does not mean that 
we can point to properties as being "exclusively due" to these environmental inputs. The innate-acquired dichotomy has been long challenged (see Lehrman 1953; Bateson and Mameli 2007) and I see no way to make Machery's distinction without a futile attempt at reifying this problematic dichotomy.

Thus, although Machery successfully dodges Hull's criticisms, the concept of human nature that he ends up proposing accords neither with intuitive notions of human nature, nor with scientific practice. It should, therefore, be discarded and be replaced. In what follows, I will propose and defend a replacement. The aim of my alternative account of human nature will be to fulfill core desiderata for such a concept. Human nature should (D1) be the empirically accessible (and thus not based on occult essences) subject of the human (psychological, anthropological, economic, biological, etc.) sciences, (D2) help clarify related concepts like innateness and naturalness, which are associated with human nature, and (D3) characterize human uniqueness. Although for some, an additional desideratum that human nature tell us something about what humans should be or should strive for is important, the notion of human nature that I offer will not directly fulfill this normative desideratum, since I hold that human nature cannot simultaneously fulfill this desideratum and D1.

\section{The life history trait cluster account of human nature. Consider an individual} human of a particular genetic constitution in a particular environment. There are many different possible outcomes to such an individual's life. Think of these possible outcomes as possible life histories. One life history involves the individual becoming relatively prosperous, having a large family, and dying after a long life. Another life history 
terminates from a fatal disease in childhood. These life histories are populated by a multitude of traits. Some of these traits persist over entire life histories (e.g., a core body temperature in excess of $90^{\circ} \mathrm{F}$ ), while others are short lived (e.g., a temperature of $105^{\circ} \mathrm{F}$ ), or momentary (a particular sneeze). 'Trait' here thus picks out any feature of a life history, no matter its duration or significance.

Now consider the totality of traits and how they are dispersed over the totality of possible life histories. It is clear that the traits do not populate the possible life histories in a random way—instead, there are patterns: Some later ("consequent") traits will always or usually follow certain prior ("antecedent" traits). The consequent trait of speaking fluent English will always be linked with antecedent exposure to spoken English. This might seem trivially true, but it is important that this is not the case with all individuals (human or otherwise). The traits can of course be morphological or physiological or psychological and not just behavioral. For individuals lacking the gene for the enzyme phenylalanine hydroxylase (PAH), those with (and only with) antecedent exposure to phenylalanine will exhibit the consequent trait of phenylketonuria (PKU), a devastating neurological disease. This rather simple observation that traits are non-randomly dispersed over this set of life histories (and that there will be robust patterns of antecedent-consequent pairs) provides the basis for the notion of individual nature, from which I will construct the concept of human nature. Individual nature is defined as the pattern of trait clusters within the individual's set of possible life histories. This concept of individual nature, though coherent, points to something that scientists will typically want to study only as a means to learning about humans in general—learning, that is, about human nature. 
Human nature is defined as the pattern of trait clusters within the totality of extant human possible life histories. Thus, if one were to take all of the possible life histories that form the basis for individual nature, and then combine them, one would possess the set of life histories that forms the basis for human nature. The trait distribution patterns in this set of life histories constitute human nature. For example, the traits "bearing offspring" and "lactating" will be clustered, the former being antecedent to the latter. There will be few instances of lactating that are not associated with the antecedent trait "bearing offspring." It is this pattern of human life history trait clusters that I am identifying with human nature.

Note that I am not arguing that these patterns are what define membership in the species. I am thus not adopting a homeostatic property cluster conception of the definition of species (see Boyd 1999). The trait patterns in an individual's nature do not determine to which species the individual belongs (though they of course serve as evidence); it is instead, as Hull and the scientific consensus argues, belonging to a particular lineage that determines species membership. This view of human nature is thus fully consistent with the cladistic view of specieshood.

This account of human nature I will label the Life-history Trait Cluster (LTC) account. This is to distinguish it from accounts that are essentialist or normative, since it is neither based on essential properties, nor, as we will see in section 7, does it imply that human nature is in any sense "good." Instead, characterizations of features of human nature are merely descriptions of statistical trends within the collective set of human life histories. 
5. Human nature: the subject of the social and psychological sciences. At first blush, the LTC account of human nature might seem to be of little use. It fails to identify a property or set of properties essential to (or good for) being human. Furthermore, by linking human nature to an infinite set of life histories, it would appear that human nature is not even empirically accessible, thus failing D1. In this section, I hope to show that, on the contrary, the LTC account identifies just what it is that is the subject of the human sciences.

To begin, let us consider what kind of results of psychological studies are of value. A study merely reporting that humans are sometimes aggressive will be of little interest. In the LTC framework, it is uninformative because it is merely calling attention to the existence of some traits within the set of human life histories, but is not identifying (or quantifying) a pattern of these traits. Such a study would never be published in an academic journal. If, instead, the study reports that adults who were abused as children will tend to be aggressive toward their own children, then the study is of interest and, if executed well, is the sort of research that could be published. What such a study is doing is identifying a pattern in the collective life histories. It is making the claim that life histories with the antecedent trait "abused as a child" will tend to be associated with the consequent trait "aggressive toward one's children."

Similarly, controlled experiments are seeking to discover life history trait patterns. A study that has participants give speeches on unfamiliar topics in front of an unfriendly audience and then measures cortisol levels in their saliva are searching for such patterns. Here a possible pattern would be an antecedent "uncomfortable public speaking event" followed by "high cortisol levels." Control groups in such studies are used to see whether 
the presence of the antecedent is causally linked to the consequent. And in the LTC framework, the controls are a way of refining our knowledge of the trait patterns and projecting into unknown possibilities. The psychologist wants to state, quite generally, that public speaking is a source of stress. And making these general statements is to say that there is a robust pattern of association between the antecedent and consequent traits. Thus, knowledge of human nature (in the LTC sense) is just the aim of psychological investigations such as these.

By extension, it is easy to see that knowledge in the human sciences more generally is, for the most part, knowledge of human nature. An anthropologist who describes an unusual behavior among the Yaminawa is going to investigate both the meaning of the behavior as well as its causes. Such an investigation is but an investigation into trait patterns — what other psychological, behavioral, physiological, etc. traits are linked to the unusual behavior? Similarly, the behavioral economist who shows that greater choice leads to poorer satisfaction is pointing to a life-history trait pattern: antecedent states "decisions with many options" will be associated with consequent states like "poor satisfaction." The degree to which the findings are robust is the degree to which there is a strong statistical association between the antecedent and consequent in the set of life histories.

Non-human animals are often used as models for studying humans. The reason why such research can be useful is also accounted for by the LTC. A model (a rat, say) in some domain (like cancer research) is going to useful to the extent that the same antecedent-consequent pattern exists in both humans and rats - cancer as consequent and extensive exposure to benzene as antecedent, for example. 
6. The quantification of human nature. We have seen that D1 is satisfied: human nature is indeed the subject of the human sciences. But it has not yet been made clear what the LTC account implies about other ways that the concept of human nature is used, i.e., it has not yet been shown to fulfill D2 (the clarification of related concepts like innateness and naturalness). We speak of a behavior being "natural," or it being part of "human nature" to behave in a particular way. Are such locutions undermined by the LTC account or can they be understood within it? In this section I will show that while it is a mistake to understand traits as dichotomously either "natural" or not, a part of "human nature" or not, I will show that there is a sense in which traits can be more or less natural, more or less central to human nature. In order to accomplish this, I will construct a human nature space and suggest that behaviors occupying a particular region are core features of human nature, while those in other parts of the space are less central.

Human nature, as argued for above, is investigated by determining associations between antecedent and consequent traits in the collective human life histories. There are two key variables that one could use in characterizing these associations. First, there is the proportion of life histories that exhibit the antecedent trait. This could also be understood as the probability that an individual drawn at random will exhibit the antecedent during their life. Second, there is the proportion of those exhibiting the antecedent who also exhibits the consequent. This can also be understood probabilistically as the conditional probability of exhibiting the consequent given the antecedent. I will label the first the pervasiveness, $p$, of the antecedent, and I will label the second the robustness, $r$, of the antecedent-consequent association. Some antecedent 
traits (lacking PAH and consuming phenylalanine) will be rare, but robustly associated with their consequent (PKU). And some antecedents can be common (imbibing alcohol) but not very robustly associated with particular consequents (like esophageal cancer), despite the fact that imbibing alcohol does raise the incidence of such cancer.

These examples exhibit two important features of the $p-r$ space. One is that the antecedent need not be a single, simple trait, but can instead be complex a trait or cluster of traits. The second is that there will generally be a tradeoff between $p$ and $r$. For a given consequent, one can often increase robustness by adding more antecedent traits (or replacing a simpler antecedent with a more complex one). Lacking the gene for PAH will be associated with PKU, but the realization that individuals can consume diets absent in phenylalanine makes the absence of PAH not all that robustly linked with PKU. However, the antecedent "lacking PAH + consuming phenylalanine" is more robustly linked to the consequent, PKU. The same is true of the alcohol example. Singling out heavy drinkers, or heavy drinkers that are also smokers, will increase the robustness of the link between the antecedent and esophageal cancer.

This tradeoff between $p$ and $r$ parallels the tradeoff that Lewis (1973) saw in the creation of deductive systems. He argued that "a contingent generalization is a law of nature if and only if it appears as a theorem (or axiom) in each of the true deductive systems that achieves a best combination of simplicity and strength" (73). Such deductive systems can generally be axiomatized more or less simply, and there is a tradeoff: "Simplicity without strength can be had from pure logic, strength without simplicity from (the deductive closure of) an almanac" (73). By making the antecedent traits more and more complex, one can increase $r$, but at a cost of decreased $p$. And one can achieve a 
high $p$ by simplifying the antecedent, but this usually comes at a cost to $r$. The analogy of Lewis's almanac is a set of antecedents that picks out the totality of antecedent traits. The consequent would have perfect robustness, but the specific set of antecedents will be singular, with the lowest possible pervasiveness.

With the $p-r$ space in mind, we are now in the position of being able to return to the question of what it might mean to "behave naturally" or for a behavior to be part of human nature. The LTC framework implies that instead of saying that it is natural to C, we should instead say that it is natural for As to C, where 'A' denotes the antecedent(s) and ' $\mathrm{C}$ ' denotes the consequent. Thus, instead of saying that it is natural to develop PKU, one should instead say that it is natural for those who (A) lack PAH and consume phenylalanine to (C) develop PKU. Similarly, instead of saying that PKU is a part of human nature, it is more informative and precise to say that it is part of human nature for individuals who lack PAH and consume phenylalanine to develop PKU. Analogously instead of stating that "lactation is a part of human nature," one could state that "lactation is a part of female human nature" or that "female lactation is a part of human nature," since the latter descriptions pick out an antecedent (being female) that makes the antecedent-consequent association robust.

If a trait is a part of human nature, then so, too, it might seem that it is innate. As discussed above, the innate-acquired distinction is problematic. But there is nevertheless a sense in which the LTC framework can provide a revised and improved way of understanding this concept. Here are three ways that one could understand innateness in the LTC framework. First, the $r$-value of the trait association is one possible way of quantifying innateness - the higher the $r$-value, the more the consequent is associated 
with the given the antecedent. Thus, it is not that consequent traits, or traits in general, that are innate full stop, but they have a quantifiable degree of innateness provided the antecedent. Second, one could restrict innateness to antecedent-consequent associations that exhibit both a high $r$-value and a high $p$-value.

Third, although these understandings of innateness harmonize with some of the standard ways of understanding the concept (in terms of canalization, for example), they do not preserve the "innateness = not learned" definition, since learning can be a part of the causes of the consequent. To preserve the "not learned" conception of innateness, one could add the restriction that learning must not be causally relevant to the appearance of the consequent, given the antecedent, though I imagine that many traits one would be apt to call innate would no longer be classified as such under this restricted definition, since learning is woven into the causal fabric of so much of development.

The LTC account of human nature thus fulfills D2 and, as we saw in the previous section, D1. But what about D3, namely, the identification of human uniqueness? And, furthermore, is there any sense in which the framework can provide insight into human goodness - what we should strive for in becoming a good human, or what we should aim for through human enhancement?

7. Human uniqueness and the question of normativity. The LTC account is incredibly permissive. All sorts of antecedent-consequent links exist, many of which are rather trivial. It is human nature for females to lactate, but this is true of all mammals. This does not mean that it is not an important feature of human nature, but it does mean that it is not uniquely human. And there are countless rather trivial trait associations. "Every human 
that has mass will die" has maximal $r$ and $p$-values, but is utterly otiose. In fact, trait associations with maximal $r$ and $p$-values will tend to be trivial. The interesting ones often occur when the antecedent is not universal, and when changes in the antecedent are causally associated with changes in the consequent. In order to both eliminate the trivial associations and to capture human uniqueness, I will define uniquely human nature as the subset of the antecedent-consequent associations that are unique to the human species. Importantly, uniquely human nature, like human nature, is a property not of each individual human, but instead of the set of extant human (actual and possible) life histories. Speaking a human language fluently (provided exposure to this language while growing up) is part of uniquely human nature. This is true because no other species will speak fluently given a similar upbringing. Raising a chimp in the same way as an American child does not result in it speaking English. The same will be true of many of the consequents that we laude in the human species, such as complex systems of morality and the ability for self-reflection.

The LTC account's "uniquely human nature" is thus a way of capturing human uniqueness, satisfying D3. But what of normativity, is there a sense in which human nature is good, or can be improved upon via human enhancement? The short answer is that because the foundations of the LTC framework are trait distribution patterns, it is, strictly speaking, descriptive and not normative. Furthermore, there is not some eternal “human nature," like a fixed target in Plato's heaven, that humans can strive for. Instead, human nature simply tracks the form, behavior, etc. of humans. Human nature was different in our species' past and will be different in the future. 
But this does not mean that there are no moral implications of human nature under the LTC framework. If the study of human nature is the study of patterns of trait associations, then studying human nature will provide insight into human goodness and evil—if a particular nefarious consequent is robustly associated with a particular set of antecedents, then the elimination or reduction of the consequent should be pursued via the elimination or alteration of one or more of the antecedents. Similarly good consequent traits can be made more common via an increase in the antecedents with which they are robustly associated.

8. Conclusions. "Man, in a word, has no nature" (Ortega y Gassett, 1961, 217, emphasis in original). Such a sentiment is shared by many in the humanities and human sciences, and seems to be based on the reflection that humans are simply too diverse (across cultures, genders, times) for there to be some human essence that we could extract from this diversity. Such skepticism is warranted if the only notion of human nature on the table is an essentialist one. But the LTC framework provides an alternative. It embraces the diversity, showing that there are patterns within and across human heterogeneity. If there is to be an empirically-accessible human nature that sheds troubling essentialisms, then it should be founded on the unique pattern of traits within the collective human life histories. Such a concept of human nature cannot play all of the roles that we may desire of it — showing us how to be more fully human, for example — but it can play many of the other roles. I have shown that it can be understood as the subject of the various human sciences, can clarify what we mean when we classify a trait as innate or natural, and can also provide a basis for human uniqueness. 


\section{References}

Bateson, P. and Mameli, M. 2007. The innate and the acquired: Useful clusters or a residual distinction from folk biology? Developmental Psychobiology, 49: 818-831

Boyd, R. 1999. Homeostasis, species, and higher taxa. In Species: New interdisciplinary essays., ed. Robert A. Wilson, 141-185MIT Press. Cambridge.

Darwin, C. 1859. On the origin of species by means of natural selection London: Murray.

Geertz, C. 1973. The interpretation of cultures Basic Books.

Hull, D. L. 1986. On human nature. Paper presented at PSA: Proceedings of the Biennial Meeting of the Philosophy of Science Association, 2: 3-13.

Lehrman, D. S. 1953. A critique of konrad lorenz's theory of instinctive behavior. The Quarterly Review of Biology 28 (4): 337-63.

Lewis, D. K. 1973. Counterfactuals Harvard University Press.

Machery, E. 2008. A plea for human nature. Philosophical Psychology 21 (3): 321-9.

y Gasset, J. O. 1961. History as a system: And other essays toward a philosophy of history WW Norton \& Company. 